Algebraic 83 Geometric $\mathcal{T}$ opology

Volume 5 (2005) 1197-1222

Published: 20 September 2005

ATG

\title{
On knot Floer homology and cabling
}

\author{
Matthew HedDen
}

\begin{abstract}
This paper is devoted to the study of the knot Floer homology groups $\widehat{H F K}\left(S^{3}, K_{2, n}\right)$, where $K_{2, n}$ denotes the $(2, n)$ cable of an arbitrary knot, $K$. It is shown that for sufficiently large $|n|$, the Floer homology of the cabled knot depends only on the filtered chain homotopy type of $\widehat{C F K}(K)$. A precise formula for this relationship is presented. In fact, the homology groups in the top 2 filtration dimensions for the cabled knot are isomorphic to the original knot's Floer homology group in the top filtration dimension. The results are extended to $(p, p n \pm 1)$ cables. As an example we compute $\widehat{H F K}\left(\left(T_{2,2 m+1}\right)_{2,2 n+1}\right)$ for all sufficiently large $|n|$, where $T_{2,2 m+1}$ denotes the $(2,2 m+1)$-torus knot.
\end{abstract}

AMS Classification 57M27; 57R58

Keywords Knots, Floer homology, cable, satellite, Heegaard diagrams

\section{Introduction}

In [8], Ozsváth and Szabó introduced a collection of abelian groups associated to closed oriented three-manifolds: given a three-manifold $Y$ and $\operatorname{Spin}^{c}$ structure $\mathfrak{s}$, there are various Heegaard Floer homology groups of $Y: \widehat{H F}(Y, \mathfrak{s})$, $H F^{\infty}(Y, \mathfrak{s}), H F^{+}(Y, \mathfrak{s})$, and $H F^{-}(Y, \mathfrak{s})$. In [12] they subsequently showed that a knot $K \subset Y$ induces a filtration on the chain complexes which compute these groups, see also [17]. In particular, the filtered chain homotopy types of the filtered chain complexes were shown to be topological invariants of the knot and the $\operatorname{Spin}^{c}$ structure. This paper will deal with the case $Y=S^{3}$ and primarily with the simplest objects defined in [12, $\widehat{H F K}(K, i)$. The notation here, as in the rest of this paper, agrees whenever possible with that of [12, 13, 11, so that the lack of reference to the three-manifold in $\widehat{H F K}(K, i)$ implies $Y=S^{3}$ and the index $i$ refers to the level of the filtration induced on $\widehat{H F}\left(S^{3}\right)$ by $K$. Specific definitions and relevant notation will be discussed in Section 2.

Recall that the $(p, q)$ cable of a knot $K$, denoted $K_{p, q}$, is defined to be the topological type of a knot supported on the boundary of a tubular neighborhood 



Figure 1: On the left is the right handed trefoil, with writhe +3 . On the right is the $(2,7)$ cable of the trefoil.

of $K$ which is linear with slope $p / q$ with respect to the standard framing of this torus. In other words, it is a satellite knot which winds $p$ times around the meridian of $K$ as it winds $q$ times around a specified longitude. This longitude is determined by the Seifert framing for $K$. The knot which is cabled is sometimes called the companion knot (see [7] for more details).

Cabling a knot increases its complexity in some sense. If one draws a projection for a knot and its $(p, p n+1)$ cable (where $n$ is the writhe of the original knot's projection), the number of crossings in the latter projection will be $p^{2}$ times the number of crossings of the original diagram plus $(p-1)$ (see Figure 1).

The Alexander polynomials of a knot and its cables are related by the following classical formula (which is a special case of a similar formula holding for all satellites):

$$
\Delta_{K_{p, q}}(t)=\Delta_{T_{p, q}}(t) \cdot \Delta_{K}\left(t^{p}\right),
$$

where $T_{p, q}$ denotes the $(p, q)$ torus knot, and $\Delta_{K}(t)$ the symmetrized Alexander polynomial of $K$ [7]. It is proved in [12 that the following relationship holds between the Euler characteristics of $\widehat{H F K}$ and the symmetrized Alexander polynomial:

$$
\sum_{i} \chi(\widehat{H F K}(K, i)) \cdot T^{i}=\Delta_{K}(T)
$$

It is therefore a natural question to ask how Equation (11) manifests itself within $\widehat{H F K}$, and, more generally, how $\widehat{H F K}$ of a knot and its satellites are related. We demonstrate some results in this direction. Before stating the first theorem, recall from [14] that $\operatorname{deg} \widehat{H F K}(K)$ denotes the largest integer $d>0$ for which 
$\widehat{H F K}(K, d) \neq 0$ and that [15] identifies this invariant with the Seifert genus of $K$. Note that also $\operatorname{deg} \widehat{H F K}\left(T_{p, q}\right)=\frac{(p-1)(q-1)}{2}$. Let us denote the filtration of $\widehat{C F}\left(S^{3}\right)$ induced by $K$ by $\mathcal{F}(K, j)$, so that we have the sequence of inclusions:

$$
0=\mathcal{F}(K,-i) \subseteq \mathcal{F}(K,-i+1) \subseteq \ldots \subseteq \mathcal{F}(K, n)=\widehat{C F}\left(S^{3}\right),
$$

with $\frac{\mathcal{F}(K, j)}{\mathcal{F}(K, j-1)}=\widehat{C F K}(K, j)$. The following theorem will be proved in Section 3 .

Theorem 1.1 Let $K$ be a knot in $S^{3}$, and suppose $\operatorname{deg} \widehat{H F K}(K)=d$. Then $\exists N>0$ such that $\forall n>N$ the following holds:

$$
\operatorname{deg} \widehat{H F K}\left(K_{2,2 n+1}\right)=2 d+n \text {. }
$$

Furthermore, $\forall i \geq 0$ we have

$$
\widehat{H F K}_{*}\left(K_{2,2 n+1}, i\right) \cong \begin{cases}H_{*+2(k-d)}(\mathcal{F}(K, k-d)) & \text { for } i=2 d+n-2 k \\ H_{*+2(k-d)+1}(\mathcal{F}(K, k-d)) & \text { for } i=2 d+n-2 k-1 .\end{cases}
$$

By the symmetry of $\widehat{H F K}$ under the involution on $\operatorname{Spin}^{c}$ structures (Equation (5) in Section (2) the above result completely determines $\widehat{H F K}\left(K_{2,2 n+1}\right)$. Note that the information required above is more than simply $\widehat{H F K}(K, i)$ for all $i$. $\widehat{H F K}(K)$ is the homology of an associated graded of a filtered chain complex - one needs to know $H_{*}(\mathcal{F}(K))$ to fully exploit the theorem. Despite this additional requirement, the theorem is still a powerful calculational tool. For instance, in 2] it is shown that the Floer homology of $(1,1)$ knots is combinatorial. They show this by exhibiting a genus one Heegaard diagram for a $(1,1)$ knot. Since the differentials in these cases can be computed combinatorially via the Riemann mapping theorem, $\widehat{H F K}$ of $(2,2 n+1)$ cables will be given combinatorially as well (for large $n$ ). Note that $(1,1)$ knots include torus knots and 2-bridge knots as a proper subset. In the case of $(p, p n+1)$ cables, we have the following result:

Theorem 1.2 Let $K, d$ be as above. Then $\exists N>0$ and $c\left(c^{\prime}, n, p\right)$ such that $\forall n>N$, the following holds:

$$
\operatorname{deg} \widehat{H F K}\left(K_{p, p n+1}\right)=p d+\frac{(p-1)(p n)}{2} .
$$

If $i>c\left(c^{\prime}, n, p\right)$ we have

$\widehat{H F K}_{*}\left(K_{p, p n+1}, i\right) \cong \begin{cases}H_{*+2(k-d)}(\mathcal{F}(K, k-d)) & \text { for } i=p d+\frac{(p-1)(p n)}{2}-p k \\ H_{*+2(k-d)+1}(\mathcal{F}(K, k-d)) & i=p d+\frac{(p-1)(p n)}{2}-p k-1 \\ 0 & \text { otherwise. }\end{cases}$

Where $c^{\prime}$ is a fixed constant coming from the projection of $K$, and $c\left(c^{\prime}, n, p\right)$ is linear in $n$ and quadratic in $p$. 
In some examples we don't know $H_{*}(\mathcal{F}(K))$. The theorem can still provide useful information if all that is known is $\widehat{H F K}(K)$ in the top filtration dimension.

Corollary 1.3 With $K, d, n>N$ as above

$$
\begin{aligned}
\operatorname{deg} \widehat{H F K}\left(K_{p, p n+1}\right) & =p \operatorname{deg} \widehat{H F K}(K)+\operatorname{deg} \widehat{H F K}\left(T_{p, p n+1}\right) \\
& =p d+\frac{(p-1)(p n)}{2} .
\end{aligned}
$$

Furthermore,

$$
\begin{aligned}
\widehat{H F K}_{*}\left(K_{p, p n+1}, p d+\frac{(p-1)(p n)}{2}\right) & \cong \widehat{H F K}_{*-1}\left(K_{p, p n+1}, p d+\frac{(p-1)(p n)}{2}-1\right) \\
& \cong \widehat{H F K}_{*}(K, d) .
\end{aligned}
$$

Of course the corollary is just the restriction of Theorem 1.2 to the top 2 filtration dimensions. However, it shows that when $\widehat{H F K}$ is successful in distinguishing knots by using only the top filtration dimension (as is the case for the Kinoshita-Terasaka knots and their Conway mutants, see [14, 6]), it also distinguishes their $(p, p n+1)$ cables. In light of [15], the corollary also shows that in many cases the Seifert genus of cabled knots is a linear function of the companion knot's genus, a result proved in [18] in general.

The proof of Theorem 1.2 relies on a special choice of Heegaard diagram for the cables of a knot which greatly simplifies their chain complexes. This diagram will be introduced in Section 2 and will subsequently be used to calculate $\widehat{H F K}\left(T_{p, q}\right)$ for some of the torus knots. With the aid of the diagram and the torus knot calculations, Theorems 1.1 and 1.2 will be proved in Section 3 Section 4 will then apply Theorem 1.1 to calculate $\widehat{H F K}$ for $(2,2 n+1)$ cables of the $(2,2 m+1)$ torus knots.

Remarks It is interesting to compare the theorems and corollary above with Equation (11). We also remark that all of the above results have corresponding analogues when $n<0$, and hence we obtain results for $(p, p n \pm 1)$ cables. These are discussed at the end of Section 3 .

Acknowledgment I cannot thank Peter Ozsváth enough for his willingness and patience to teach me the subject and his support and enthusiasm as my advisor. 


\section{Preliminaries, Heegaard diagrams, and useful Ex- amples}

\subsection{Preliminaries on knot Floer homology}

Let $K \subset S^{3}$ be a knot. In 12, Ozsváth and Szábo introduced the knot Floer complex $\widehat{C F K}(K)=\bigoplus_{i \in \mathbb{Z}} \widehat{C F K}(K, i)$ associated to a Heegaard diagram for a knot, and whose homology groups are knot invariants, see also [17, 13. This complex depends upon a suitable choice of Heegaard diagram, compatible with the knot in the following sense:

Definition 2.1 A compatible doubly-pointed Heegaard diagram for a knot $\mathrm{K}$ (or simply a Heegaard diagram for $\mathrm{K}$ ) is a collection of data

$$
\left(\Sigma,\left\{\alpha_{1}, \ldots, \alpha_{g}\right\},\left\{\beta_{1}, \ldots, \beta_{g-1}, \mu\right\}, w, z\right),
$$

where

- $\Sigma$ is an oriented surface of genus g

- $\left\{\alpha_{1}, \ldots, \alpha_{g}\right\}$ are pairwise disjoint, linearly independent embedded circles which specify a handlebody, $U_{\alpha}$, bounded by $\Sigma$

- $\left\{\beta_{1}, \ldots, \beta_{g-1}, \mu\right\}$ are pairwise disjoint, linearly independent embedded circles which specify a handlebody, $U_{\beta}$, bounded by $\Sigma$ such that $U_{\alpha} \cup_{\Sigma} U_{\beta}$ is diffeomorphic to $S^{3}$

- If we do not attach the handle specified by $\mu$, together with the final three-ball necessary to make $S^{3}$, then the resulting three-manifold with boundary is the knot complement, $S^{3} \backslash \nu(K)$ (i.e. $\mu$ is the meridian of the knot)

- The points $z$ and $w$ can be joined by a small arc $\delta$, oriented from $z$ to $w$, which intersects none of $\left\{\alpha_{1}, \ldots, \alpha_{g}, \beta_{1}, \ldots, \beta_{g-1}\right\}$ and algebraically intersects $\mu$ the same number as $l k(K, \mu)$ if we arbitrarily orient $\mu$.

We now briefly recall the definitions of $\widehat{C F K}(K)$ and its boundary operator in terms of this diagram, though the reader unfamiliar with the subject is strongly encouraged to read Section 2 of [13]. While [12] sets up the machinery for knot Floer homology in a more general context, the level of generality here will be consistent with that of [13]. For this reason all definitions and notation used here are consistent with those of [13] unless otherwise specified. 
Recall that the knot Floer homology is a doubly graded homology theory. One grading is a homological grading (coming from the grading on the Floer homology of $S^{3}$ ), also called the Maslov grading. The other grading comes from a filtration of $\widehat{C F}\left(S^{3}\right)$ induced by the knot. This latter grading will be referred to as the filtration or $\operatorname{Spin}^{c}$ grading. The chain complex $\widehat{C F K}$ is generated by intersection points of the tori $\mathbb{T}_{\alpha}=\alpha_{1} \times \ldots \times \alpha_{g}$ and $\mathbb{T}_{\beta}=\beta_{1} \times \ldots \times \beta_{g-1} \times \mu$ contained in $\operatorname{Sym}^{g}\left(\Sigma_{g}\right)$. Any two such points can be connected by a Whitney disk $\phi$ whose boundary is contained in the tori. We denote the intersection number of $\phi$ with the submanifold $p \times \operatorname{Sym}^{g-1}\left(\Sigma_{g}\right)$ by $n_{p}(\phi)$, where $p$ is any point in $\Sigma-\alpha_{1}-\ldots-\alpha_{g}-\beta_{1}-\ldots-\beta_{g-1}-\mu$.

The relative Maslov and $\operatorname{Spin}^{c}$ gradings (denoted gr and $\mathcal{F}$ respectively) are determined by the following (found in [13]):

$$
\begin{aligned}
& \operatorname{gr}(\mathbf{x})-\operatorname{gr}(\mathbf{y})=\mu(\phi)-2 n_{w}(\phi) \\
& \mathcal{F}(\mathbf{x})-\mathcal{F}(\mathbf{y})=n_{z}(\phi)-n_{w}(\phi),
\end{aligned}
$$

where $\mu$ is the Maslov index of $\phi$. The absolute Maslov grading is obtained by the convention that $\operatorname{gr}(\mathbf{x})=0$ for $\mathbf{x}$ generating $\widehat{H F}\left(S^{3}\right) \cong \mathbb{Z}$. The absolute filtration grading can be naively obtained by requiring $\widehat{H F K}(K, i)$ to be symmetric about $i=0$, though it has a more invariant description given in [12].

The boundary operator $\partial_{z}$ is defined as follows:

$$
\partial_{z}[\mathbf{x}]=\sum_{\mathbf{y} \in \mathbb{T}_{\alpha} \cap \mathbb{T}_{\beta}} \sum_{\left\{\phi \in \pi_{2}(\mathbf{x}, \mathbf{y}) \mid \mu(\phi)=1, n_{w}(\phi)=0\right\}} \#(\widehat{\mathcal{M}}(\phi))[\mathbf{y}],
$$

where here $\widehat{\mathcal{M}}(\phi)$ denotes the quotient of the moduli space of $J$-holomorphic disks representing the homotopy type of $\phi, \mathcal{M}(\phi)$, divided out by the natural action of $\mathbb{R}$ on this moduli space. This operator can act on various chain complexes: $\widehat{C F}\left(S^{3}\right), \mathcal{F}(K, j), \widehat{C F K}(K, j)$, for example. The resulting homologies will be denoted $\widehat{H F}\left(S^{3}\right), H(\mathcal{F}(K, j))$, and $\widehat{H F K}(K, j)$. The operator decomposes as a sum $\partial_{z}=\partial_{z}^{0}+\partial_{z}^{1}+\ldots+\partial_{z}^{k}$ where $\partial_{z}^{i}$ has the same formula as above except we require $n_{z}(\phi)=i$ in addition to $n_{w}(\phi)=0$. We define $\partial_{w}$ with the same formula except we require $n_{z}(\phi)=0$. Similarly $\partial_{w}^{i}$ requires $n_{z}(\phi)=0$ and $n_{w}(\phi)=i$.

\section{$2.2 \quad$ A Heegaard diagram for cables}

The purpose of this section is to demonstrate an appropriate Heegaard diagram for the $(p, p n+1)$ cable of a knot $K$. The following lemma describes a procedure 
for finding a Heegaard diagram for cable knots starting from a diagram for the pattern. Note that, strictly speaking, the diagram we obtain for the cable is not actually compatible in the sense of Definition 2.1 since it does not contain the meridian of the cable as a $\beta$ attaching curve. However, a compatible diagram can easily be obtained by stabilizing the diagram described below. Due to the independence of Heegaard Floer homology under (de)stabilization [8], we simply work with the diagram for the cable obtained below. For more details on the Heegaard diagrams used in this paper see Chapter 2 of [4].

Lemma 2.2 Let $\left(\Sigma,\left\{\alpha_{1}, \ldots, \alpha_{g}\right\},\left\{\beta_{1}, \ldots, \beta_{g-1}, \mu\right\}, z, w\right)$, be a Heegaard diagram for a knot $K$. Then a Heegaard diagram for $K_{p, p n+1}$ is obtained from the diagram for $K$ by replacing $\mu$ with a curve $\tilde{\beta}$. The curve $\tilde{\beta}$ is obtained by winding $\mu$ along an $n$-framed longitude for the knot $(p-1)$ times. The point $w$ is to remain fixed under this operation. The point $z$ is replaced by a basepoint $z^{\prime}$ so that the arc connecting $z^{\prime}$ and $w$ has algebraic intersection number $p$ with $\tilde{\beta}$ and is disjoint from all other $\beta$ curves and all $\alpha$ curves. (See Figures 3 and 4 .)

Proof Let us first understand the Heegaard diagram for $K_{2,1}$ in terms of the Heegaard diagram for K. Begin with the unknot. A Heegaard diagram for the unknot is simply the standard Heegaard diagram for $S^{3}$, together with two points, $z$ and $w$, placed a small distance apart on either side of the curve $\mu$ representing the meridian, Figure 2A.

Now draw in place of the unknot its $(2,1)$ cable. Of course this is still the unknot. However, $\mu$ is no longer a meridian for the knot. It has $l k(K, \mu)=2$. Thus we stabilize the diagram in the sense of [3] by drilling a hole between the strands of the knot. When stabilizing, we add two curves to the diagram, $\alpha^{\prime}$ and $\mu^{\prime}$, which bound disks when their corresponding handles are attached, and which satisfy $\alpha^{\prime} \cap \mu^{\prime}=1$. The curve $\mu^{\prime}$ (which does not encircle the added hole) can be chosen so that it is a meridian for the cabled unknot. On either side of this meridian we add the points $z^{\prime}$ and $w$ (we add the prime here and throughout to signify that this is a diagram for the cabled knot) in such a way to be compatible with the orientation of the knot. See Figure 2B. Although the diagram with no modification to the original $\alpha$ and $\mu$ curves represents $S^{3}$, it is not a Heegaard diagram for the knot since $\mu$ links the knot twice. Thus we replace $\mu$ by a curve $\beta$ whose attaching disk does not intersect the knot, and which still results in a Heegaard diagram for $S^{3}$. The attaching disk of $\beta$ stays between the two strands of the knot, twisting as the knot twists, while it winds along the longitude of the original unknot. See Figure 2C. Now we perform two 
A

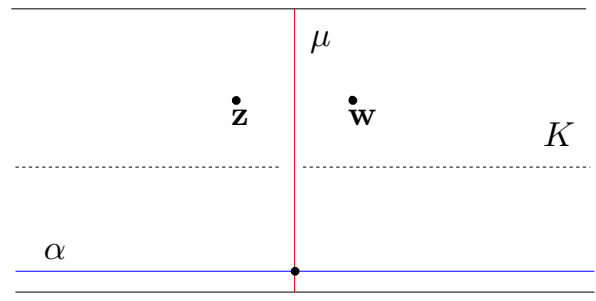

C

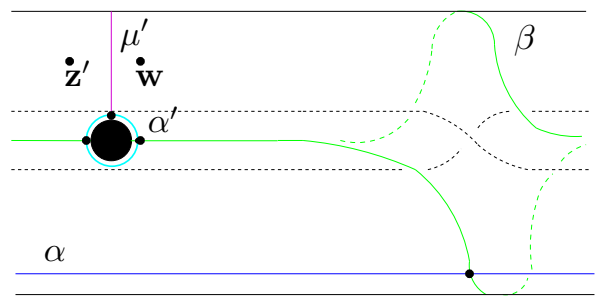

$\mathrm{B}$

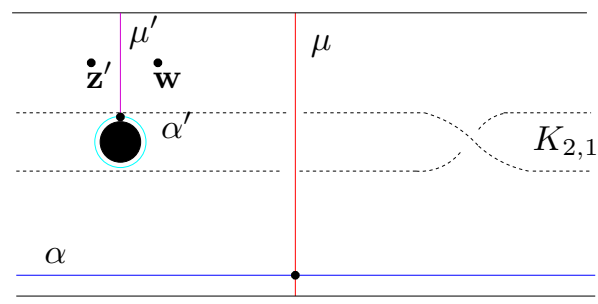

$\mathrm{D}$

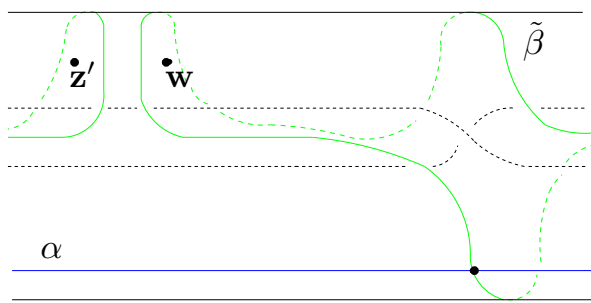

Figure 2: Illustration of Lemma 2.2 The four figures represent the steps of the lemma. Each is a top view of a region of the solid torus. A dashed line represents either the knot in the core of the solid torus, or an attaching circle for the Heegaard diagram which is on the underside of the torus. The dark circle in figures $\mathrm{B}$ and $\mathrm{C}$ is a hole drilled through the torus. It is destabilized in D after two handleslides.

handleslides on $\beta$ to obtain a curve $\tilde{\beta}$ which satisfies the requirements of the lemma and no longer intersects $\alpha^{\prime}$. We can destabilize the resulting diagram, removing $\mu^{\prime}$ and $\alpha^{\prime}$,to obtain a genus one diagram for the $(2,1)$ cable of the unknot satisfying the conditions of the lemma. See Figure 2 D.

To find a Heegaard diagram for the $(2,2 n+1)$ cable of an arbitrary knot we note that our choice of the unknot above was not special: the same sequence of Heegaard moves could have been applied with an arbitrary knot in a handlebody of genus $g$. The index, $n$, of the cable corresponds to the framing of the knot used in the Heegaard diagram. If we use the zero framing, the procedure yields the $(2,1)$ cable as above. Picking a framing which adds $n$ meridians to the 0 -framed longitude for $K$ is equivalent to cabling with the $(2,2 n+1)$ torus knot.

Finally, to obtain a diagram for the $(p, p n+1)$ cable of a knot, it is easy to see (if somewhat harder to draw) that the above procedure can be extended. The only difference is that instead of winding the original meridian once along the 
longitude, we wind it $(p-1)$ times (while still winding $n$ times in the meridional direction.)

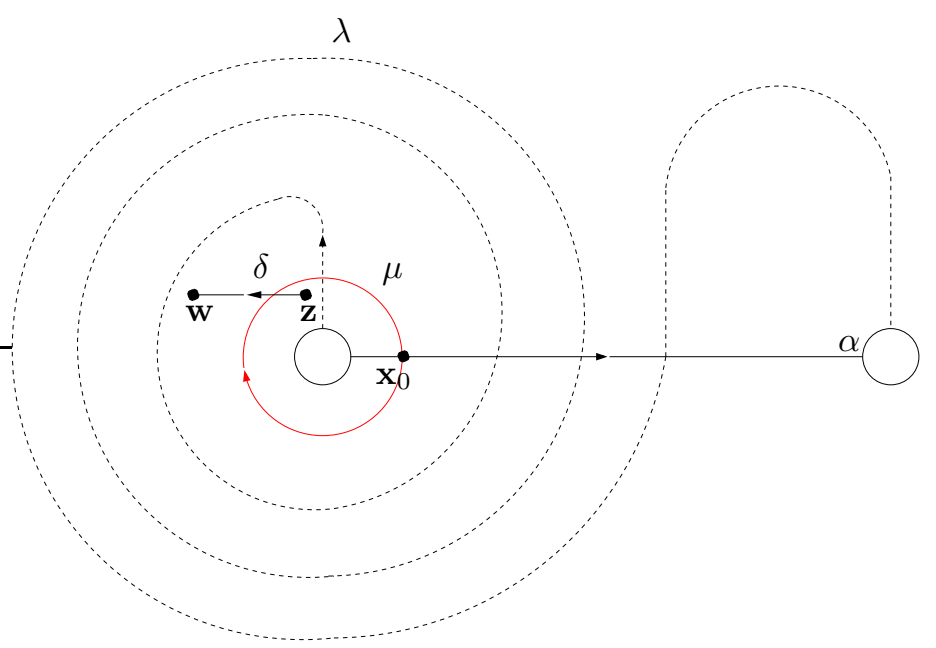

Figure 3: Heegaard diagram for the unknot. $\lambda$ represents a 3 -framed longitude for the unknot around which we will wind the meridian, $\mu$.

\subsection{Examples: $\widehat{H F K}\left(T_{2,2 n+1}\right), \widehat{H F K}\left(T_{3,7}\right)$}

As both an illustration of Lemma 2.2 and also as a tool for the general case, we calculate $\widehat{H F K}$ for the $(2,2 n+1)$ and $(3,7)$ torus knots. As it turns out, much of what we see in these simple examples is reflected in the cables of an arbitrary knot when $n$ is large.

\section{Proposition 2.3}

$$
\widehat{H F K}\left(T_{2,2 n+1}, i\right) \cong \begin{cases}\mathbb{Z}_{(i-n)} & \text { for }|i| \leq n \text { if } n \geq 0 \\ \mathbb{Z}_{(i-n-1)} & \text { for }|i| \leq-n-1 \text { if } n<0 .\end{cases}
$$

The groups in both cases vanish outside of the specified range for $i$.

Of course this result is known, 9]. (For generalizations, see Theorem 1.3 of [13], Theorem 1.2 of [1], or [17.) Nonetheless it will be interesting to see the result arise in this context.

Proof To obtain a Heegaard diagram for $T_{2,2 n+1}$, we start with the standard genus one diagram for the unknot and apply Lemma 2.2 using an $n$-framed 


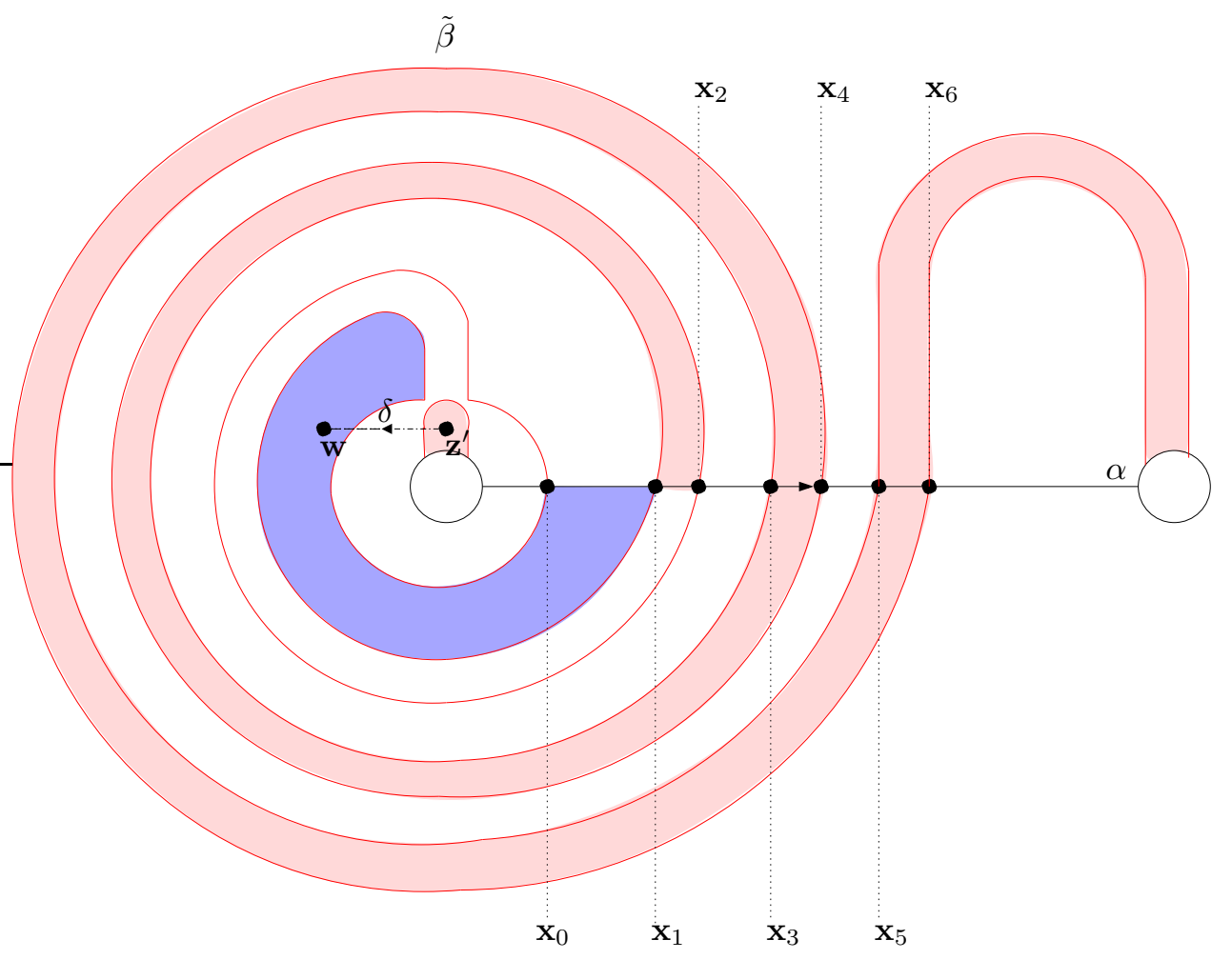

Figure 4: Heegaard diagram for the $(2,7)$ cable of the unknot (i.e. $\left.T_{2,7}\right)$ obtained from the diagram of the unknot above via Lemma 2.2 $\delta$ has intersection 2 with $\tilde{\beta}$. The darkly shaded (blue) region indicates the domain of the differential $\phi$ connecting $\mathbf{x}_{1}$ to $\mathbf{x}_{0}$ discussed in the proof of Proposition 2.3 which has $n_{w}(\phi)=1$. The lightly shaded (red) region indicates the domain of the differential $\psi$ connecting $\mathbf{x}_{1}$ to $\mathbf{x}_{2}$ and which has $n_{z^{\prime}}(\psi)=1$

longitude. This process is pictured in Figures 3 and 4. From Figure 4 we see there are $2 n+1$ intersection points. To determine the intersection point which has Maslov grading zero, we disregard the basepoint $z^{\prime}$ in the diagram. Now we are free to isotope $\tilde{\beta}$ back around the longitude, removing all intersection points but $\mathbf{x}_{0}$. Thus $\mathbf{x}_{0}$ generates $\widehat{H F}\left(S^{3}\right) \cong \mathbb{Z}$ and has Maslov grading zero.

We claim that for $n>0, \operatorname{gr}\left(\mathbf{x}_{i}\right)-\operatorname{gr}\left(\mathbf{x}_{i+1}\right)=\mathcal{F}\left(\mathbf{x}_{i}\right)-\mathcal{F}\left(\mathbf{x}_{i+1}\right)=1$. This follows immediately from the fact that for $i$ odd there is a unique holomorphic disk $\phi$ connecting $\mathbf{x}_{i}$ to $\mathbf{x}_{i-1}$ which has $n_{w}(\phi)=1$ and $n_{z^{\prime}}(\phi)=0$, and also a unique holomorphic disk $\psi$ connecting $\mathbf{x}_{i}$ to $\mathbf{x}_{i+1}$ which has $n_{w}(\psi)=0$ and $n_{z^{\prime}}(\psi)=1$. Examples of these disks are shown in Figure 4. The statement about the Maslov and filtration gradings follows from Equations (3) and (4) 
above.

For $n \geq 0$, the proposition follows immediately by noting that each intersection point lives in a distinct filtration level. The case for $n<0$ is completely analogous, except that $\operatorname{gr}\left(\mathbf{x}_{i}\right)-\operatorname{gr}\left(\mathbf{x}_{i+1}\right)=\mathcal{F}\left(\mathbf{x}_{i}\right)-\mathcal{F}\left(\mathbf{x}_{i+1}\right)=-1$ since the winding occurs in the opposite direction. Be careful to note that using a -1 framed longitude to obtain a Heegaard diagram for $T_{2,-1}$ actually produces a Heegaard diagram with 3 intersection points (when one would expect it to have 1 , since it is the unknot). However, these additional intersection points can be removed using an allowed isotopy of $\tilde{\beta}$ (i.e. one that does not cross $z^{\prime}$ or $w$.) Indeed, whenever $n<0$ we can remove two intersection points in this way.

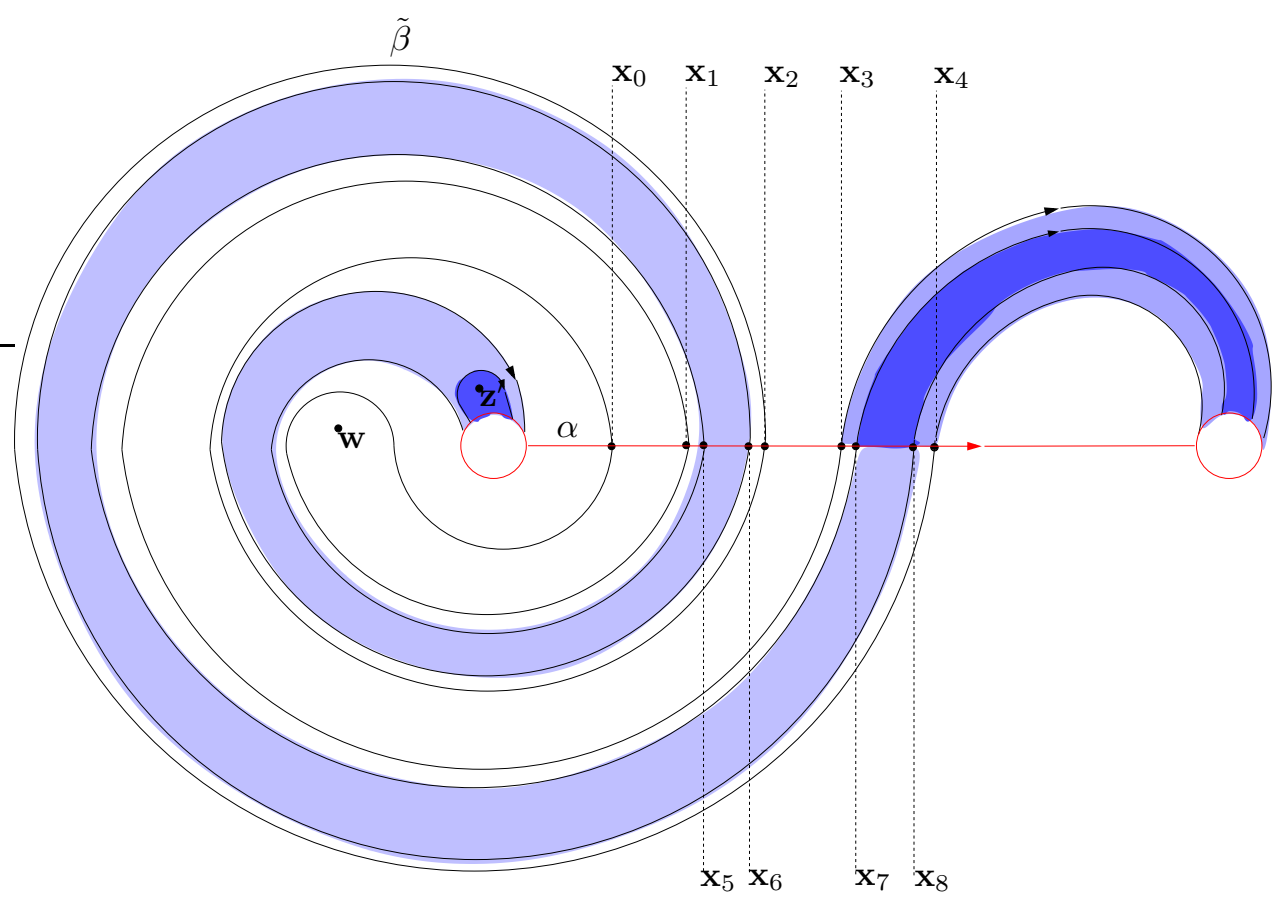

Figure 5: Heegaard diagram for the $(3,7)$ cable of the unknot (i.e. $\left.T_{3,7}\right)$ obtained via Lemma 2.2 with $p=3, n=2$ The shaded region indicates the domain $\phi$ connecting $\mathbf{x}_{3}$ to $\mathbf{x}_{4}$ discussed in the proof of Proposition 2.4 The light shading indicates multiplicity 1 while the dark shading multiplicity 2

In the interest of being concrete, rather than extend the above example to the case of the torus knots $T_{p, p n+1}$, we calculate only a specific example, $T_{3,7}$. The case of $T_{p, p n+1}$ follows in exactly the same spirit as $T_{3,7}$ and is only notationally more difficult. In general, $\widehat{H F K}\left(T_{p, q}\right)$ follows from Theorem 1.2 of [1]. 


\section{Proposition 2.4}

$$
\widehat{H F K}\left(T_{3,7}, i\right) \cong \begin{cases}\mathbb{Z}_{(0)} & i=6 \\ \mathbb{Z}_{(-1)} & i=5 \\ \mathbb{Z}_{(-2)} & i=3 \\ \mathbb{Z}_{(-3)} & i=2 \\ \mathbb{Z}_{(-4)} & i=0 \\ 0 & i=1,4, i>6\end{cases}
$$

Remark The knot Floer homology groups enjoy a symmetry under the natural involution on $\operatorname{Spin}^{c}$ structures given by conjugation, Proposition 3.10 of [12. For the purposes of this paper, this symmetry can be expressed by the following formula:

$$
\widehat{H F K}_{*}(K, i) \cong \widehat{H F K}_{*-2 i}(K,-i) \text {. }
$$

Thus the information in the above proposition completely specifies $\widehat{H F K}\left(T_{3,7}\right)$.

Proof We apply Lemma 2.2 with $p=3, n=2$, to obtain the diagram for $T_{3,7}$ shown in Figure 5. There are nine intersection points $\mathbf{x}_{0} \ldots \mathbf{x}_{8}$. Just as in the proof of Proposition 2.3, there are unique holomorphic disks with domains $\phi$ having $n_{w}(\phi)=1, n_{z^{\prime}}(\phi)=0$ which connect $\mathbf{x}_{1}$ to $\mathbf{x}_{0}$ and $\mathbf{x}_{3}$ to $\mathbf{x}_{2}$. Both of these domains have $\mu(\phi)=1$. There are obvious holomorphic disks connecting $\mathbf{x}_{1}$ to $\mathbf{x}_{2}$ and $\mathbf{x}_{3}$ to $\mathbf{x}_{4}$ with $n_{z^{\prime}}(\phi)=2, n_{w}(\phi)=0$ and $\mu(\phi)=1$ (see Figure 5). The last two obvious holomorphic disks connect $\mathbf{x}_{5}$ to $\mathbf{x}_{6}$ and $\mathbf{x}_{7}$ to $\mathbf{x}_{8}$ with $n_{z^{\prime}}(\phi)=1, n_{w}(\phi)=0$, and $\mu(\phi)=1$.

We now calculate the relative filtration difference between $\mathbf{x}_{4}$ and $\mathbf{x}_{6}$. Let $A$ and $B$ be a symplectic basis for $H_{1}\left(\Sigma_{1}, \mathbb{Z}\right)$ such that $[B] \cdot[A]=+1$ and so that $A=\alpha$ and $[B]=[\tilde{\beta}]$ (assuming we orient the curves as in Figure 5). Draw an arc from $\mathbf{x}_{4}$ to $\mathbf{x}_{6}$ along $\alpha$ and an arc from $\mathbf{x}_{6}$ to $\mathbf{x}_{4}$ following $\beta$. The result is a closed curve $\gamma$ which can be chosen so that $[\gamma] \cdot[A]=1$ and $[\gamma] \cdot[B]=1$. Hence,

$$
[\gamma-B-A]=[\gamma-\tilde{\beta}-\alpha]=0 \in H_{1}\left(\Sigma_{1}, \mathbb{Z}\right) .
$$

Thus there is a null-homology $\phi$ for the curve $\gamma-\tilde{\beta}-A$ which has $n_{w}(\phi)=0$. $n_{z^{\prime}}(\phi)$ is then the algebraic intersection number of an arc $\delta$ connecting $z^{\prime}$ to $w$, with $\gamma-\tilde{\beta}-A$. Equivalently, it is the multiplicity of the null-homology constructed at the point $z^{\prime}$. We see from this that $n_{z^{\prime}}(\phi)=3$ and hence that $\mathcal{F}\left(\mathbf{x}_{4}\right)-\mathcal{F}\left(\mathbf{x}_{6}\right)=n_{z^{\prime}}(\phi)-n_{w}(\phi)=3-0=3$. A similar analysis shows that $\mathcal{F}\left(\mathbf{x}_{5}\right)-\mathcal{F}\left(\mathbf{x}_{7}\right)=3$. Together with the statements above, we see that each point is in a different filtration dimension and hence generates a $\mathbb{Z}$ summand in 
the associated graded homology. Requiring $\mathrm{rk} \widehat{H F K}(i)$ to be symmetric about $i=0$ yields the statement of the groups in the proposition.

To find the Maslov grading of the groups we argue similarly to Proposition 2.3. If we forget the reference point $z^{\prime}$ and slide $\tilde{\beta}$ back, we find that $\mathbf{x}_{0}$ must have absolute grading 0 . The theorem then follows from Equations (3) and (5) together with the Maslov indices of the disks in the first paragraph.

\section{Proof of theorems}

In this section we prove the theorems stated in the introduction. The idea behind these theorems is the following: when we perform the operation of Lemma 2.2 the resulting Heegaard diagram can be simultaneously viewed as a diagram for both the original and cabled knot by appropriately placing three basepoints. When we increase $n$ we add a large number of spirals to the Heegaard diagram. If we make this spiraling region large enough, we can ensure that all generators in the complex for both the original and cabled knots having high filtration gradings live in the spiraling region. Since the domains of the differentials in the Heegaard diagram are the same regardless of whether we are viewing it as a diagram for the original or cabled knot, we can use our assumed knowledge of the original knot's differential to calculate the differential for the cabled knot. When we specialize to the case of $(2,2 n+1)$ cables, the symmetry of $\widehat{H F K}$ under the conjugation action on $\operatorname{Spin}^{c}$ structures (Equation (5D)) allows us to completely determine $\widehat{H F K}\left(K_{2,2 n+1}\right)$. With the idea in place, we begin.

\subsection{Proof of Theorems 1.2 and 1.1}

Given a $g$-bridge presentation for $K$, we obtain a genus $g$ Heegaard diagram for $S^{3}$ compatible with $K$ (see [3]). See Figure 6 for the Heegaard diagram of the right-handed trefoil. Note that the meridian for the knot will intersect $\alpha_{g}$ exactly once in the point $x_{0}$, and will intersect none of the other $\alpha$ curves. This ensures that all intersection points of the knot's chain complex will be of the form $\left(x_{0}, \mathbf{y}\right)$ for some $(g-1)$ tuple, $\mathbf{y}$, of intersection points. Following [12] one can determine the filtration grading of these generators. For each filtration summand, $\widehat{C F K}(K, i)$, there is a unique set of intersection points generating the summand. Let us denote by $C(i)$ all $g-1$ tuples of intersection points between $\mathbb{T}_{\beta} \backslash \mu$ and $\mathbb{T}_{\alpha} \backslash \alpha_{g}$ which, together with $x_{0} \in \alpha_{g} \cap \mu$, generate $\widehat{C F K}(K, i)$. Thus we write $x_{0} \times C(i)$ to mean $\widehat{C F K}(K, i)$. 
Apply Lemma 2.2. The first thing to note is that by appropriately placing a third basepoint, $z$, the diagram can be viewed as compatible either with $K$ or with $K_{p, p n+1}$ :

Lemma 3.1 Let $z$ be a point on the arc $\delta$ connecting $z^{\prime}$ to $w$ for which the segment of $\delta$ connecting $z$ to $w$ has intersection number 1 with $\tilde{\beta}$. See Figure 7. Then the Heegaard diagram with the pair $\left(w, z^{\prime}\right)$ is compatible with $K_{p, p n+1}$, while with the pair $(w, z)$ it is compatible with $K$.

Proof That the diagram with $\left(w, z^{\prime}\right)$ is compatible with $K_{p, p n+1}$ is just Lemma 2.2. To see that the diagram with $(w, z)$ is compatible with $K$, simply isotope $\tilde{\beta}$ in the reverse direction to that of Lemma 2.2 in order to arrive at the original diagram for $K$. For the diagram with $(w, z)$ this is an allowed isotopy in the sense of [12] since it does not cross either basepoint.

Let $n \gg 0$. This creates a large spiraling region in the Heegaard diagram, similar to the diagrams for the torus knots in Section 2. This region contains an odd number, $2(p-1) n+1$, of intersection points of $\tilde{\beta}$ with $\alpha_{g}$. Denote these intersection points $x_{0} \ldots x_{2(p-1) n}$. See Figure 5 or 7 . (One should be careful here. If we start with a 0 -framed longitude and apply Lemma 2.2 there will be at least $2(p-1) n+1$ intersection points, but possibly more. The 0 framed longitude for $K$ may intersect $\alpha_{g}$ ) When we look at $x_{i} \times C(j)$ we find that there is a "copy" of each filtration summand of the original knot's chain complex carried by the point $x_{i}$. In light of this we define:

Definition 3.2 In the Heegaard diagram for the $(p, p n+1)$ cable of $K$ we call an intersection point an exterior intersection point if it is of the form $\left(x_{i}, \mathbf{y}\right)$ where $x_{i} \in \tilde{\beta} \cap \alpha_{g}$ and $x_{i}$ can be joined to $x_{0}$ by an arc which intersects $\tilde{\beta}$ geometrically at most $2(p-1) n-1$ times and which intersects none of the other attaching curves. All other intersection points will be called interior.

The exterior points are those that arise from the spiraling region. Before continuing further we establish a labeling convention for the $\alpha_{g} \cap \tilde{\beta}$ component of the exterior points. We label these points as follows: each time we wind $\tilde{\beta}$ around the longitude (i.e. increase the parameter $p$ ) we add $2 n$ intersection points $x_{i} \in \alpha_{g} \cap \tilde{\beta}$ generating exterior points. First we label the points that arise the first time we wind around the longitude. From left to right we label them $x_{0} \ldots x_{2 n}$. If $p=2$, we are done. If $p>2$ we next label the points arising the second time we wind around the longitude $x_{2 n+1}, \ldots, x_{4 n}$, again from left 
to right, and so on. See Figure 5 for a picture of the spiraling region with this labeling convention.

The exterior intersection points play a primary role in the proof of the theorem. If we make $n$ large enough, the chain complexes in highest filtration dimensions (with respect to the filtrations induced by both the uncabled and cabled knot) will be generated by a subset of the exterior points. To see this, we must understand the relative filtrations induced by $K$ and $K_{p, p n+1}$. We develop this knowledge through a sequence of lemmas. Let us denote the filtration with respect to $z$ (i.e. induced by $K$ ) by $\mathcal{F}$ and the filtration with respect to $z^{\prime}$ (i.e. induced by $\left.K_{p, p n+1}\right)$ by $\mathcal{F}^{\prime}$.

We begin with the relative filtration between exterior points sharing the same $(g-1)$-tuple, $\mathbf{y}$.

Lemma 3.3 For $i<2 n$ odd, we have

$$
\begin{gathered}
\mathcal{F}\left(x_{i-1}, \mathbf{y}\right)-\mathcal{F}\left(x_{i}, \mathbf{y}\right)=\mathcal{F}^{\prime}\left(x_{i-1}, \mathbf{y}\right)-\mathcal{F}^{\prime}\left(x_{i}, \mathbf{y}\right)=1 \\
\mathcal{F}\left(x_{i}, \mathbf{y}\right)-\mathcal{F}\left(x_{i+1}, \mathbf{y}\right)=0 \\
\mathcal{F}^{\prime}\left(x_{i}, \mathbf{y}\right)-\mathcal{F}^{\prime}\left(x_{i+1}, \mathbf{y}\right)=p-1
\end{gathered}
$$

Proof For $i$ odd, there is a holomorphic disk with domain $\phi$ from $\left(x_{i}, \mathbf{y}\right)$ to $\left(x_{i-1}, \mathbf{y}\right)$ having $n_{z}(\phi)=n_{z^{\prime}}(\phi)=0, n_{w}(\phi)=1$. It is the product of the disk from Propositions 2.3 and 2.4 (connecting $\mathbf{x}_{i}$ to $\mathbf{x}_{i-1}$ ) with the constant map in $\operatorname{Sym}^{g-1}\left(\Sigma_{g}\right)$. There is a disk with domain $\psi$ from $\left(x_{i}, \mathbf{y}\right)$ to $\left(x_{i+1}, \mathbf{y}\right)$ with $n_{z}(\psi)=n_{w}(\psi)=0, n_{z^{\prime}}(\psi)=p-1$. This is the the analogue of the disk in Propositions 2.3 and 2.4 connecting $\mathbf{x}_{i}$ to $\mathbf{x}_{i+1}$. Topologically it is still a disk, but now it wraps around the longitude $p-1$ times. The lemma follows from Equation (4).

Next we fix $x_{i}$ and vary the $(g-1)$-tuple.

Lemma 3.4 Suppose $\mathbf{y} \in C(j), \mathbf{z} \in C(k)$. Then,

$$
\begin{gathered}
\mathcal{F}\left(x_{i}, \mathbf{y}\right)-\mathcal{F}\left(x_{i}, \mathbf{z}\right)=j-k \\
\mathcal{F}^{\prime}\left(x_{i}, \mathbf{y}\right)-\mathcal{F}^{\prime}\left(x_{i}, \mathbf{z}\right)=p(j-k) .
\end{gathered}
$$

Proof The two $(g-1)$-tuples had filtration difference $j-k$ in the original Heegaard diagram by assumption (i.e. before we applied Lemma 2.2). Thus the boundary of the domain connecting these points in the original diagram had intersection number $j-k$ with an arc connecting $z$ and $w$. In the cabled 
diagram, the boundary of the new domain will still have intersection number $j-k$ with the arc connecting $z$ to $w$ while it will have intersection number $p(j-k)$ with the arc connecting $z^{\prime}$ and $w$. See Figures [6] and 7 for an illustration of this lemma.

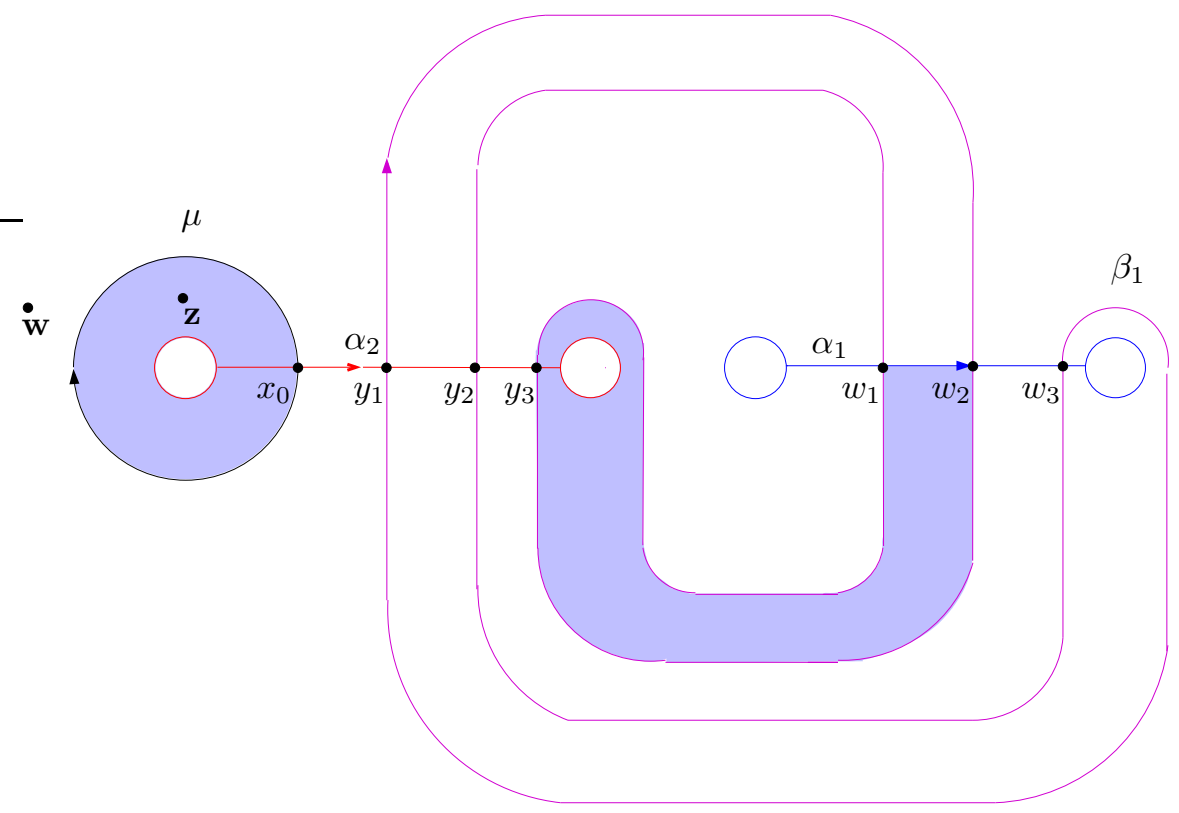

Figure 6: Heegaard diagram for the right-handed trefoil coming from its 2-bridge presentation. Shaded is a domain $\phi$ connecting $\left(x_{0}, w_{2}\right)$ to $\left(x_{0}, w_{1}\right)$ having $n_{z}(\phi)=1$, $n_{w}(\phi)=0$. When we apply Lemma 2.2 this domain winds along the longitude and the resulting domain $\phi^{\prime}$ has $n_{z^{\prime}}\left(\phi^{\prime}\right)=p, n_{z}\left(\phi^{\prime}\right)=1$, and $n_{w}\left(\phi^{\prime}\right)=0$. See Figure 7 for an illustration when $p=2$.

We now know the relative filtration grading for both the cabled and uncabled knot of all intersection points of the form $\left(x_{i}, \mathbf{y}\right)$, with $i \leq 2 n$. See Figure 8 for a table depicting the two chain complexes.

There are many more intersection points in general $-\tilde{\beta}$ intersects the other $\alpha$ curves as well as $\alpha_{g}$. See Figure 7 for an example of these intersection points. We must also understand the filtration grading of the points $\left(x_{i}, \mathbf{y}\right)$ when $i>2 n$ (the rest of the exterior points). These points occur when $p>2$ because $\tilde{\beta}$ winds more than once around the longitude for $K$. To this end we have the following:

Lemma 3.5 There exist constants $N, c^{\prime}>0$ such that $\forall n$ with $n>N$, the relative filtration gradings of the exterior intersection points containing 


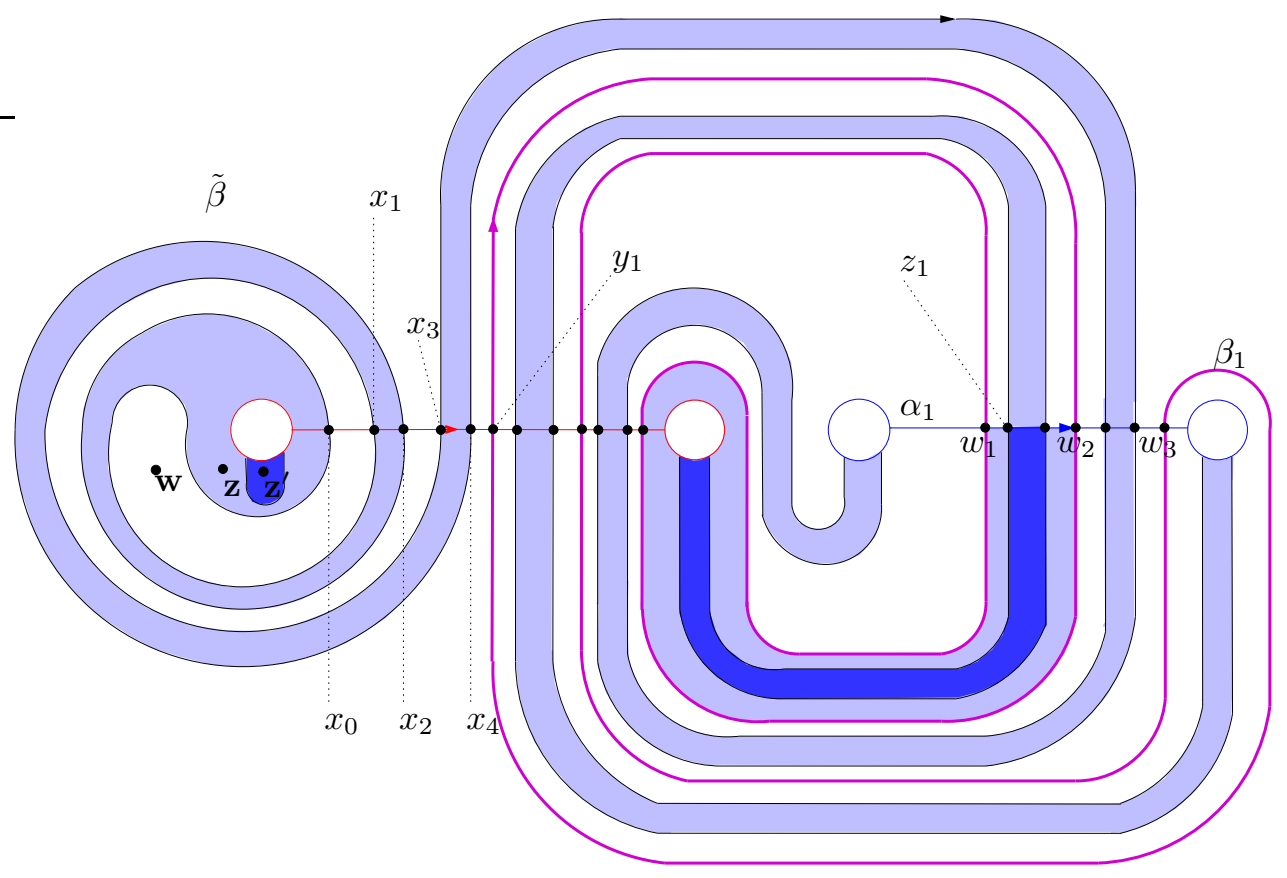

Figure 7: Heegaard diagram for the $(2,13)$ cable of the right-handed trefoil. The exterior intersection points are those of the form $\left(x_{i}, w_{j}\right)$ for $i=0, \ldots, 4, j=1,2,3$ (there are only 5 points $x_{i} \in \alpha_{2} \cap \tilde{\beta}$ generating exterior points because the others were removed by an isotopy of $\tilde{\beta}$ not crossing $\mathbf{z}^{\prime}$.) Note the domain $\phi^{\prime}$ from $\left(x_{i}, w_{2}\right)$ to $\left(x_{i}, w_{1}\right)$ for $i=0, \ldots, 4$. This is the domain from Figure 6 which was wound along the longitude to have $n_{z^{\prime}}\left(\phi^{\prime}\right)=2$. The point $\left(y_{1}, z_{1}\right)$ is an example of an interior point.

$x_{0} \ldots x_{2\left(n-c^{\prime}\right)}$ are higher than the filtration grading of all other intersection points except possibly those containing $x_{2\left(n-c^{\prime}\right)+1} \ldots x_{2 n}$. The $n$, as always, refers to the parameter specifying the cabled knot $K_{p, p n+1}$. The constant $c^{\prime}$ is independent of $n$ and depends only on the projection of $K$.

Proof First note that the relative filtration difference between any interior points, $\mathbf{p}$ and $\mathbf{q}$, does not change as we vary $n$. This is because domains connecting interior points either remain fixed (take place entirely in the interior of the diagram) or simply add area to the part of the domain with multiplicity in the spiral. In either case $n_{z^{\prime}}(\phi), n_{z}(\phi), n_{w}(\phi)$ all remain fixed.

Next observe that the relative filtration difference between points of the form $\left(x_{2 n}, \mathbf{y}\right)$ and all the interior points is fixed as we vary $n$. The reason is the same as above: Domains connecting $\left(x_{2 n}, \mathbf{y}\right)$ to the interior at worst change by adding 


\begin{tabular}{|c|c|c|c|}
\hline & $C(d)$ & $C(d-1)$ & $C(d-2)$ \\
\hline$x_{0}$ & $(0,0)$ & $(-1,-p)$ & $(-2,-2 p)$ \\
\hline$x_{1}$ & $(-1,-1)$ & $(-2,-p-1)$ & $(-3,-2 p-1)$ \\
\hline$x_{2}$ & $(-1,-p)$ & $(-2,-2 p)$ & $(-3,-3 p)$ \\
\hline$x_{3}$ & $(-2,-p-1)$ & $(-3,-2 p-1)$ & $(-4,-3 p-1)$ \\
\hline$x_{4}$ & $(-2,-2 p)$ & $(-3,-3 p)$ & $(-4,-4 p)$ \\
\hline$\vdots$ & $\vdots$ & $\vdots$ & $\vdots$ \\
\hline$x_{2 n}$ & $(-n,-n p)$ & $(-1-n,-n p-p)$ & $(-2-n,-n p-2 p)$ \\
\hline
\end{tabular}

Figure 8: Table of relative filtrations of exterior points for $K$ and $K_{p, p n+1}$ when $n>N$. The columns and rows are arranged to distinguish the elements $\left(x_{i}, \mathbf{y}\right) \in x_{i} \times C(j)$. The number on the left is the relative filtration of the points in each summand taken with respect to $z$ (the original knot's basepoint). The number on the right is the relative filtration of the points taken with respect to $z^{\prime}$ (the cabled knot's basepoint). The dashed box is the chain complex for the cabled knot in relative filtration $-p$. It is contained in the solid box of generators with relative $z$ filtration greater than -2 . Proposition 3.7 shows that the complex in the dashed box is actually a subcomplex of the complex in the solid box. Note the filtration gradings here are relative.

area to the part of the domain in the spiral and hence $n_{z^{\prime}}(\phi), n_{z}(\phi), n_{w}(\phi)$ are all constant.

Finally we must account for the other exterior points. Let $i>0$. We need to calculate the filtration difference between $x_{2 n}$ and $x_{2 n+i}$. We claim that both $\mathcal{F}\left(x_{2 n}\right)-\mathcal{F}\left(x_{2 n+i}\right)$ and $\mathcal{F}^{\prime}\left(x_{2 n}\right)-\mathcal{F}^{\prime}\left(x_{2 n+i}\right)$ are bounded below by some constant $\mathrm{K}$, independent of $n$.

To prove the claim observe that $\mathcal{F}\left(x_{2 n}\right)-\mathcal{F}\left(x_{2 n+1}\right)$ and $\mathcal{F}^{\prime}\left(x_{2 n}\right)-\mathcal{F}^{\prime}\left(x_{2 n+1}\right)$ are independent of $n$ for the same reasons as above. The homological method for calculating filtration differences of Proposition 2.4 shows that when $n$ is large enough, $\mathcal{F}\left(x_{2 n+1}\right)-\mathcal{F}\left(x_{2 n+j}\right)$ and $\mathcal{F}^{\prime}\left(x_{2 n+1}\right)-\mathcal{F}^{\prime}\left(x_{2 n+j}\right)$ are both always greater than or equal to zero for any $j>1$. These observations, the previous two lemmas, and the fact that there are only a finite number of interior intersection points proves the lemma. 
We now wish to prove:

Lemma 3.6 For $n>N$ as above,

$$
\begin{gathered}
\widehat{H F K}_{*}\left(K_{p, p n+1}, \text { topmost }\right) \cong \widehat{H F K}_{*-1}\left(K_{p, p n+1}, \text { topmost }-1\right) \cong \widehat{H F K}_{*}(K, d) \\
\widehat{H F K}\left(K_{p, p n+1}, \text { topmost }-i\right) \cong 0 \text { for } i=2, \ldots, p-1,
\end{gathered}
$$

Where topmost refers to the highest filtration dimension for which $\widehat{H F K}\left(K_{p, p n+1}\right) \neq 0$ and $d=\operatorname{deg} \widehat{H F K}(K)$.

Proof When $n>N$ the above Lemmas show that the exterior points $x_{0} \times C(d)$ are higher in relative filtration than all other intersection points. This holds whether we take our filtration with respect to $z$ (the original knot) or $z^{\prime}$ (its cable). It follows from this and Equation (4) that the domain of any disk connecting points of the form $x_{0} \times C(d)$ with $n_{w}=0$ must have $n_{z}=n_{z^{\prime}}=0$ as well. This implies that the differential restricted to these points is independent of the basepoint used and we immediately have $\widehat{H F K}\left(K_{p, p n+1}\right.$, topmost $) \cong$ $\widehat{H F K}(K, d)$. We do not yet know the absolute filtration grading for the cable and hence we simply refer to the dimension as "topmost" for now. The gradings are the same for either knot because the chain complex calculates $\widehat{H F K}\left(S^{3}\right)$ regardless of which way it is filtered. Thus a generating point for this homology is independent of the filtration. Furthermore, the relative Maslov grading is calculated using Equation (3), which is also independent of the filtration used. This proves that the first and last groups stated in the lemma are isomorphic.

We show the first and second groups are isomorphic. Recall that $\partial_{w}$ decomposes as a sum $\partial_{w}=\partial_{w}^{0}+\partial_{w}^{1}+\ldots+\partial_{w}^{k}$, where $\partial_{w}^{i}$ is the boundary operator counting holomorphic disks whose domains have $n_{z}(\phi)=0$ and $n_{w}(\phi)=$ $i$. $\quad\left(\partial_{w}\right)^{2}=0$ implies $\partial_{w}^{1}$ is a chain map from $\widehat{C F K}\left(K_{p, p n+1}\right.$, topmost- 1$)$ to $\widehat{C F K}\left(K_{p, p n+1}\right.$, topmost). For each (g-1) tuple $\mathbf{y}$, there is an obvious holomorphic disk with domain $\phi$ connecting $\left(x_{1}, \mathbf{y}\right)$ to $\left(x_{0}, \mathbf{y}\right)$ with $n_{z}(\phi)=n_{z^{\prime}}(\phi)=$ $0, n_{w}(\phi)=1$. It is the product, $u \times \mathbf{y}$, of the disk $u$ in the torus from Propositions 2.3 and 2.4 with the constant map in $\operatorname{Sym}^{g-1}\left(\Sigma_{g}\right)$. We denote this summand in $\partial_{w}^{1}$ by $l_{0}$. In the standard way (see, for instance, Theorem 4.1 of [12] ) we filter the chain map $\partial_{w}^{1}$ with respect to negative area of the domains of disks. With respect to this filtration,

$$
\partial_{w}^{1}=l_{0}+\text { lower order terms. }
$$

$l_{0}$ is clearly an isomorphism of chain complexes, and hence $\partial_{w}^{1}$ induces an isomorphism of groups. The grading shift is a consequence Equation (3). 
From Lemma 3.5 and Figure 8 it is clear that $\widehat{H F K}\left(K_{p, p n+1}\right.$, topmost $\left.-i\right) \cong 0$ for $i=2, \ldots, p-1$ : there are simply no intersection points in these filtration dimensions.

Proposition 3.7 Let $K$ be a knot in $S^{3}$, and suppose that $\operatorname{deg} \widehat{H F K}(K)=d$. Then $\exists N>0$ such that for all $n>N$, the following holds:

$$
\operatorname{deg} \widehat{H F K}\left(K_{p, p n+1}\right)=p d+\frac{(p-1)(p n)}{2}
$$

Furthermore, $\exists c\left(c^{\prime}, n, p\right)$ such that if $i>c\left(c^{\prime}, n, p\right)$ we have

$\widehat{H F K}_{*}\left(K_{p, p n+1}, i\right) \cong \begin{cases}H_{*}\left(C\left(K, i^{\prime} \geq d-k\right), \partial_{w}\right) & \text { for } i=p d+\frac{(p-1)(p n)}{2}-p k \\ \widehat{H F K}_{*+1}\left(K_{p, p n+1}, i+1\right) & i=p d+\frac{(p-1)(p n)}{2}-p k-1 \\ 0 & \text { otherwise }\end{cases}$

Where $C\left(K, i^{\prime} \geq d-k\right)$ is the chain complex generated by points with filtration dimension $\geq d-k$ with respect to $K . c\left(c^{\prime}, n, p\right)$ is linear in $n$ and quadratic in $p$.

Proof The base case being established by Lemma 3.6 we assume the theorem holds for $k-1$, with $k<n-c^{\prime}$ (here $c^{\prime}$ is the constant from Lemma 3.5). It is immediate that for $k<n-c^{\prime}$

$$
\left.\widehat{H F K}_{*}\left(K_{p, p n+1}, \text { topmost }-p k\right)\right) \cong \widehat{H F K}_{*-1}\left(K_{p, p n+1}, \text { topmost }-p k-1\right) .
$$

$\partial_{w}^{1}$ induces an isomorphism of these groups just as in Lemma 3.6. We will establish the absolute filtration dimension at the end. We first note that $\left(C\left(K, i^{\prime} \geq d-k\right), \partial_{w}\right)$ is a subcomplex of $\left(\widehat{C F K}(K), \partial_{w}\right)$. This follows from the fact that $\partial_{w}$ counts only those disks whose domains have $n_{z}(\phi)=0$. Thus the domain of any differential with range outside of $\left(C\left(K, i^{\prime} \geq d-k\right), \partial_{w}\right)$ must have negative multiplicity by Equation (4). This is impossible by Lemma 3.2 of 8 .

The generators of $\widehat{C F K}\left(K_{p, p n+1}\right.$, topmost $\left.-p k\right)$ are clearly contained in $\left(C\left(K, i^{\prime} \geq d-k\right), \partial_{w}\right)$, see Figure 8 . We will show that the former is actually a subcomplex of the latter. The differential on $\widehat{C F K}\left(K_{p, p n+1}\right.$, topmost $\left.-p k\right)$ counts disks whose domains have $n_{z^{\prime}}(\phi)=n_{w}(\phi)=0$, while $\left(C\left(K, i^{\prime} \geq d-k\right), \partial_{w}\right)$ requires $n_{z}(\phi)=0$. When restricted to points in $\widehat{C F K}\left(K_{p, p n+1}\right.$, topmost $\left.-p k\right)$, however, both differentials are identical - the relative filtration gradings of generators in this set are the same with respect to either basepoint. Thus Equation (4) implies that both differentials compute the same homology, $\widehat{H F K}\left(K_{p, p n+1}\right.$, topmost $\left.-p k\right)$. 
To show that $\widehat{C F K}\left(K_{p, p n+1}\right.$, topmost $\left.-p k\right)$ is a subcomplex is to show that there are no differentials from

$$
\widehat{C F K}\left(K_{p, p n+1}, \text { topmost }-p k\right) \quad \text { to } \frac{\left(C\left(K, i^{\prime} \geq d-k\right), \partial_{w}\right)}{\widehat{C F K}\left(K_{p, p n+1}, \text { topmost }-p k\right)} .
$$

This is an application of Equation (4). The numbers on the left in Figure 8 depend on Equation (4) using $z$, while the numbers on the right use $z^{\prime}$. If

$$
\mathbf{x} \in \widehat{C F K}\left(K_{p, p n+1}, \text { topmost }-p k\right) \text { and } \mathbf{y} \in \frac{\left(C\left(K, i^{\prime} \geq d-k\right), \partial_{w}\right)}{\widehat{C F K}\left(K_{p, p n+1}, \text { topmost }-p k\right)},
$$

then

$$
\mathcal{F}(\mathbf{x})-\mathcal{F}(\mathbf{y})>\mathcal{F}^{\prime}(\mathbf{x})-\mathcal{F}^{\prime}(\mathbf{y})
$$

This immediately implies the domain connecting $\mathbf{x}$ to $\mathbf{y}$ has negative multiplicity (since $\left.n_{z}(\phi)=0\right)$. Again Lemma 3.2 of 8 implies there are no holomorphic representatives of these domains and hence $\widehat{C F K}\left(K_{p, p n+1}\right.$, topmost $\left.-p k\right)$ is a subcomplex.

The proposition, filtration dimensions aside, follows immediately - the quotient complex has trivial homology since $\partial_{w}^{1}$ induces an isomorphism between $\widehat{H F K}_{*}\left(K_{p, p n+1}\right.$, topmost $\left.-p(k-1)\right)$ and $\widehat{H F K}_{*}\left(K_{p, p n+1}\right.$, topmost $\left.-p(k-1)-1\right)$.

Calculating the absolute filtration dimension follows from what we have proved and Equations (11) and (2). The knots $K$ and $K_{p, p n+1}$ have isomorphic groups in top filtration dimension. If the Euler characteristic of this group is non-zero, then so is the coefficient of $T^{\mathcal{F} \text { (topmost) }}$ in the Alexander polynomial for both knots, and the result follows from Equation (11). If the Euler characteristic of the group is zero, proceed to the first group with non-zero Euler characteristic. One must exist since the Alexander polynomial of a knot cannot be zero. Inspection of the relative filtration levels for both knots shows that if $\operatorname{deg} \widehat{H F K}(K)-$ $\operatorname{deg} \Delta_{K}(t)=l$, then $\operatorname{deg} \widehat{H F K}\left(K_{p, p n+1}\right)-\operatorname{deg} \Delta_{K_{p, p n+1}}=p \cdot l$. This completes the proposition.

The constant $c\left(c^{\prime}, n, p\right)$ in the proposition is explained as follows: Lemma 3.5 shows that for $n>N$, the exterior points carried by $x_{0}, \ldots, x_{2\left(n-c^{\prime}\right)}$ are higher in filtration dimension than all points except those carried by $x_{2\left(n-c^{\prime}\right)+1}, \ldots, x_{2 n}$, with $c^{\prime}>0$ independent of $n$. It follows that the exterior points $\left(x_{i}, \mathbf{y}\right)$ with $i<2 n$ generate the top $p\left(n-c^{\prime}\right)+1$ filtration dimensions. Since $\operatorname{deg} \widehat{H F K}\left(K_{p, p n+1}\right)=p d+\frac{(p-1)(p n)}{2}$ we see that the constant $c\left(c^{\prime}, n, p\right)=$ $p d+\frac{(p-1)(p n)}{2}-p\left(n-c^{\prime}\right)-1$ takes the appropriate form. 
Proof of Theorems 1.1 and 1.2 Theorem 1.2 is a restatement of Proposition [3.7. The chain complex $\left(C\left(K, i^{\prime} \geq d-k\right), \partial_{w}\right)$ is naturally identified with $\mathcal{F}(-K, k-d)$, where $-K$ denotes $K$ with the reverse orientation - the differential on $\mathcal{F}(-K)$ is $\partial_{w}$ by definition, while the relative filtration equation (Equation (4)) for $-K$ permutes $z$ and $w$, see 12. The homology of $\mathcal{F}(-K, k-d)$ is identified with $\mathcal{F}(K, k-d)$ in Section 3.5 of [12].

The grading shift occurs for the following reason: the relative gradings for both the original and cabled knots are defined using

$$
\operatorname{gr}(\mathbf{x})-\operatorname{gr}(\mathbf{y})=\mu(\phi)-2 n_{w}(\phi) .
$$

The proof of Proposition 3.7, however, identifies the knot Floer homology groups of the cable with the homology of the complexes, $\left(C\left(K, i^{\prime} \geq d-k\right), \partial_{w}\right)$. Using the differential, $\partial_{w}$, the relative grading equation is

$$
\operatorname{gr}(\mathbf{x})-\operatorname{gr}(\mathbf{y})=\mu(\phi)-2 n_{z}(\phi) .
$$

The grading shift is a consequence of this and the relative filtrations.

In the special case $p=2$, we see that the constant $c\left(c^{\prime}, n, p\right)$ is negative for sufficiently large $n$, thus proving Theorem 1.1 .

Remark In the case where $n<0$, the above discussion carries through almost verbatim. The exterior points can be isolated in filtration, the only difference being that they are lower (rather than higher) in filtration grading than all other points. In addition, two exterior points can be removed by an isotopy. The proof of Proposition 3.7 is exactly the same in this setting, with the roles of subcomplex and quotient complex reversed (i.e. the cabled knot group is naturally a quotient complex rather than a subcomplex, and the first term in the short exact sequence of chain complexes has trivial homology). We state the analogue of Theorem 1.2 with $n<0$ for completeness.

Theorem 3.8 Let $K \subset S^{3}$ be a knot with deg $\widehat{H F K}(K)=d$. Then $\exists N<0$ and $c\left(c^{\prime}, n, p\right)$ such that $\forall n<N$, the following holds:

$$
\operatorname{deg} \widehat{H F K}\left(K_{p, p n+1}\right)=p d+\frac{(p-1)(p|n|-2)}{2} .
$$

If $i<-c\left(c^{\prime}, n, p\right)$ we have

$$
\widehat{H F K}_{*}\left(K_{p, p n+1}, i\right) \cong \begin{cases}H_{*+2(d-k)}\left(\frac{\widehat{C F K}(K)}{\mathcal{F}(K, d-k-1)}\right) & i=p k-p d-\frac{(p-1)(p|n|-2)}{2} \\ H_{*+2(d-k)-1}\left(\frac{\overline{\mathcal{F}}(K, d-k-1)}{\mathcal{F}(K)}\right) & i=p k+1-p d-\frac{(p-1)(p|n|-2)}{2} \\ 0 & \text { otherwise. }\end{cases}
$$

When $p=2$ we can arrange that $c\left(c^{\prime}, n, p\right)<0$. 
Proposition 3.7 of [12] relates the Floer homology of a knot $K$, and its mirror, $\bar{K}$. Thus the above theorem, together with Theorem 1.2 gives the values of $\widehat{H F K}\left(K_{p, p n \pm 1}, i\right)$ for all sufficiently large $|n|$ with $|i|>c\left(c^{\prime}, n, p\right)$.

\section{Examples}

Let $T_{2,2 m+1}$ be the $(2,2 m+1)$ torus knot. For all $i \geq 0$ We have the following:

Proposition 4.1 If $m>0,0 \leq k<m$, then for all $n>10 m$

$$
\widehat{H F K}\left(\left(T_{2,2 m+1}\right)_{2,2 n+1}, i\right) \cong \begin{cases}\mathbb{Z}_{-2 k} & \text { for } i=2 m+n-4 k \\ \mathbb{Z}_{-2 k-1} & \text { for } i=2 m+n-4 k-1 \\ 0 & \text { for } i=2 m+n-4 k-2 \\ 0 & \text { for } i=2 m+n-4 k-3 \\ \mathbb{Z}_{i-n} & \text { for } 0 \leq i \leq n-2 m \\ 0 & \text { otherwise }\end{cases}
$$

If $m<0,0 \leq k<|m|$, then for all $n>6|m|+1$

$$
\widehat{H F K}\left(\left(T_{2,2 m-1}\right)_{2,2 n+1}, i\right) \cong \begin{cases}\mathbb{Z}_{2|m|-4 k} & i=2|m|+n-4 k \\ \mathbb{Z}_{2|m|-4 k-1} & i=2|m|+n-4 k-1 \\ \mathbb{Z}_{2|m|-2 k-1} \oplus \mathbb{Z}_{2|m|-4 k-2} & i=2|m|+n-4 k-2 \\ \mathbb{Z}_{|| m \mid-2 k-2} \oplus \mathbb{Z}_{2|m|-4 k-3} & i=2|m|+n-4 k-3 \\ \mathbb{Z}_{i-n} & \text { for } 0 \leq i \leq n-2|m| \\ 0 & \text { otherwise }\end{cases}
$$

If $m>0,0 \leq k<m$, then for all $n<-6 m-1$

$$
\widehat{H F K}\left(\left(T_{2,2 m+1}\right)_{2,2 n-1}, i\right) \cong \begin{cases}\mathbb{Z}_{-2 m+4 k} & i=-2 m-|n|+4 k \\ \mathbb{Z}_{-2 m+4 k+1} & i=-2 m-|n|+4 k+1 \\ \mathbb{Z}_{-2 m+2 k+1} \oplus \mathbb{Z}_{-2 m+4 k+2} & i=-2 m-|n|+4 k+2 \\ \mathbb{Z}_{-2 m+2 k+2} \oplus \mathbb{Z}_{-2 m+4 k+3} & i=-2 m-|n|+4 k+3 \\ \mathbb{Z}_{i+|n|} & \text { for } 0 \geq i \geq 2 m-|n| \\ 0 & \text { otherwise }\end{cases}
$$

If $m<0,0 \leq k<|m|$, then for all $n<-10|m|$

$$
\widehat{H F K}\left(\left(T_{2,2 m-1}\right)_{2,2 n-1}, i\right) \cong \begin{cases}\mathbb{Z}_{2 k} & \text { for } i=-2|m|-|n|+4 k \\ \mathbb{Z}_{2 k+1} & \text { for } i=-2|m|-|n|+4 k+1 \\ 0 & \text { for } i=-2|m|-|n|+4 k+2 \\ 0 & \text { for } i=-2|m|-|n|+4 k+3 \\ \mathbb{Z}_{i+|n|} & \text { for } 0 \geq i \geq 2|m|-|n| \\ 0 & \text { otherwise }\end{cases}
$$


Proof When $n>0$ this follows directly from Proposition 2.3 and Theorem 1.1. When $n<0$ we use Theorem [3.8. Alternatively, we could use Proposition 3.7 of [12]. (In Proposition 2.3] we only remarked upon the existence of certain differentials in the chain complex for $S^{3}$. Indeed, it is not difficult to see that these are the only differentials. Use Equations (3) and (44), for instance, together with the fact that domains of holomorphic disks must be positive.)

The bounds for $n$ when $n>0, m>0$ or $n<0, m<0$ arise as follows: In the Heegaard diagram for the $(2,2 m+1)$ torus knot coming from its 2-bridge presentation, the natural longitude has framing $n=4 m$ (by natural we mean the longitude which intersects $\alpha_{2}$ geometrically the minimum number of times). Applying Lemma 2.2 with this longitude yields one point, $x_{0} \in \tilde{\beta} \cap \alpha_{2}$. It is a straightforward homological computation to see that when $m>0$ (resp. $m<0), x_{0}$ carries one intersection point, $x_{0} \times w_{2 m+1}$, with relative filtration grading higher (resp. lower) than all other points. Furthermore, the lowest (resp. highest) relative filtration dimension of any intersection point in the diagram is $12|m|$ less (resp. more) than $x_{0} \times w_{2 m+1}$. Thus the total breadth of relative filtration agrees with the breadth of the Alexander polynomial and is equal to $12|m|+1$. By the lemmas in Section 3, increasing (resp. decreasing) the framing of the longitude by $6|m|$ increases the total breadth in filtration by $12|m|$. Thus the total breadth in filtration is $24|m|+1$ whereas the breadth in filtration of the exterior points is $12|m|+1$. Therefore we may apply Theorem 1.1 (resp. 3.8). The bound in either case is $4 m+6 m=10 m$.

We determine the bound when $n<0, m>0$ or $n>0, m<0$ as follows: begin as above with the natural $4 m$-framed longitude for the $(2,2 m+1)$ torus knot. If $m>0$ (resp. $m<0$ ), the point $x_{0} \times w_{1}$ carried by $x_{0}$ with lowest (resp. highest) relative filtration is $4 m$ lower (resp. higher) in filtration than $x_{0} \times w_{2 m+1}$. This follows from Lemma 3.4. Since the total breadth in filtration is $12|m|+1$, we see that $x_{0} \times w_{1}$ is $8|m|$ higher (resp. lower) in filtration than the lowest (resp. highest) filtration dimension. Decrease (resp. increase) the framing of the longitude by 1 . This has the effect of making the total breadth of filtration $12 m$, rather than $12 m+1$. Furthermore, $x_{0} \times w_{1}$ is now $8|m|-1$ higher (resp. lower) than the lowest (resp. highest) filtration dimension. This is because a pair of points $x_{1}, x_{2} \in \tilde{\beta} \cap \alpha_{2}$ can be removed by an allowed isotopy as in Proposition 2.3. The net effect of the change in framing is moving the basepoint $w$ across $\tilde{\beta}$. Decrease (resp. increase) the framing by $4|m|$. This ensures that $x_{0} \times w_{1}$ is lower in relative filtration than all other points, interior or exterior. The framing used is now -1 (resp. 1) and the total breadth of filtration is $12|m|+1$. Decrease (resp. increase) the framing by $6|m|+1$. The exterior points isolated in filtration from the interior now account for more than 
half of the total filtration breadth. The bounds follow.

Remark It is likely that the bound here, and indeed in Theorem 1.1. can be taken to be $N=2 g(K)$. Some support of this can be found in Chapter 3 of [4, where we explicitly compute all $(2,2 n+1)$ cables of the trefoil knot.

\section{References}

[1] G Burde, H Zieschang, Knots, de Gruyter Studies in Mathematics 5, Walter de Gruyter \& Co. Berlin (1985) MathReview

[2] H Goda, H Matsuda, T Morifuji Knot Floer Homology of (1,1)-Knots arXiv:math.GT/0311084

[3] RE Gompf, A I Stipsicz, 4-manifolds and Kirby calculus, Graduate Studies in Mathematics 20, American Mathematical Society, Providence, RI (1999) MathReview

[4] M Hedden On knot Floer homology and cabling, PhD.thesis, Columbia University (2005)

[5] L H Kauffman, Formal knot theory, Mathematical Notes 30, Princeton University Press, Princeton, NJ (1983) MathReview

[6] S Kinoshita, H Terasaka, On unions of knots, Osaka Math. J. 9 (1957) 131153 MathReview

[7] W B R Lickorish, An introduction to knot theory, Graduate Texts in Mathematics 175, Springer-Verlag, New York (1997) MathReview

[8] P Ozsváth, Z Szabó, Holomorphic disks and topological invariants for closed three-manifolds, Ann. of Math. (2) 159 (2004) 1027-1158 MathReview

[9] P Ozsváth, Z Szabó, Holomorphic disks and three-manifold invariants: properties and applications, Ann. of Math. (2) 159 (2004) 1159-1245 MathReview

[10] P Ozsváth, Z Szabó, Knot Floer homology and the four-ball genus, Geom. Topol. 7 (2003) 615-639 MathReview

[11] P Ozsváth, Z Szabó, On knot Floer homology and lens space surgeries, arXiv:math.GT/0303017

[12] P Ozsváth, Z Szabó, Holomorphic disks and knot invariants, Adv. Math. 186 (2004) 58-116 MathReview

[13] P Ozsváth, Z Szabó, Heegaard Floer homology and alternating knots, Geom. Topol. 7 (2003) 225-254 MathReview

[14] P Ozsváth, Z Szabó, Knot Floer homology, genus bounds, and mutation, Topology Appl. 141 (2004) 59-85 MathReview 
[15] $\mathbf{P}$ Ozsváth, $\mathbf{Z}$ Szabó, Holomorphic disks and genus bounds, Geom. Topol. 8 (2004) 311-334 MathReview

[16] J A Rasmussen, Floer homology of surgeries on two-bridge knots, Algebr. Geom. Topol. 2 (2002) 757-789 MathReview

[17] J A Rasmussen, Floer homology and knot complements, PhD thesis, Harvard University (2003)

[18] T Shibuya, Genus of torus links and cable links, Kobe J. Math. 6 (1989) 37-42 MathReview

Department of Mathematics, Princeton University

Princeton, NJ 08544-1000, USA

Email: mhedden@math.princeton.edu

Received: 9 August 2004 Revised: 23 July 2005 